\title{
Influence of built-in obstacles on unconfined vapor cloud explosion
}

Dong Li, Qi Zhang*, Qiuju Ma, Shilei Shen, Jiachen Chen, Shaoyun Ren

State Key Laboratory of Explosion Science and Technology, Beijing Institute of Technology, Beijing 100081, China

Abstract: The obstacle structure in the vapor cloud has a significant influence on the gas explosion. Obstacles could not only lead to the acceleration of flame, but also they may occupy some space, thus affecting the amount of combustible gas. In this paper, a new two-step method was proposed to respectively study the effects of the obstacles amount and volume blockage ratio (VBR) on the gas explosion by using Computation Fluid Dynamic software AutoReaGas, and the obstacles in the vapor cloud were set to "Solid" instead of "Subgrid". Based on the results and analysis, it is found that the peak overpressure and the maximum combustion rate rise with the increase of the number of obstacles for a single VBR, which indicated that the vapor cloud explosion of more obstacles was more dangerous for a single VBR. However, under a single number of obstacles, the peak overpressure and the maximum combustion rate increase firstly and then decrease as VBR increases and reach the highest at the VBR of 0.74 , which indicated that the intensity of vapor cloud explosion reach a peak at a certain VBR in the middle instead of the largest. In addition, the existence and structure of obstacles have little effect on the size of explosion fireball when the size and concentration of combustible gas cloud are the same.

Keywords: Gas explosion; Combustible gas cloud; Obstacles amount; Obstacle volume blockage; Peak overpressure; Maximum combustion rate

\section{Introduction}

In the chemical and petroleum industries, it is almost impossible to avoid the leakage of gas and

* Corresponding author. Tel.: +8610 68914252; Fax: +86 1068914252.

E-mail address: qzhang@bit.edu.cn (Q. Zhang). 
highly volatile liquid. When a great quantity of leaking combustible gas or vapor of liquid fuel mixed together with air and reached to the threshold concentration, it may induce gas-phase explosion when encountering ignition source accidentally, thereby, causing huge casualties and property losses. This phenomenon is usually called the unconfined vapor cloud explosion (UVCE) (Bjerketvedt et al., 1997). The unconfined vapor cloud explosion accident has become one of the main forms of explosions disasters.

Most combustible gas cloud explosion accidents happened in a congested environment. The obstacles existing in vapor cloud played an important role in gas explosion. Both the obstacles amount and the obstacle volume blockage ratio (VBR) are very important factors. VBR refers to the volume ratio of the obstacles and the combustible gas. The most important aspect determining the course of a gas explosion in a complex geometry are the development of turbulence and the corresponding increase in the combustion rate during the explosion. Arntzen (1998) analysed and presented new models for modeling of turbulent reactive flows for CFD simulation of gas explosions in complex geometries like offshore modules. The turbulence models have been improved to handle transient, subgrid and reactive flows with $k-\varepsilon$ turbulence model and subgrid model. AutoReaGAS User Manual Version 3.1 (2002) presented the Navier-Stokes processor for gas explosion modeling and thought that the essence of a gas explosion consisted of the interaction of a premixed combustion process with its self-induced expansion flow field and the development of this process is predominantly controlled by the turbulence induced in the flow field by the boundary conditions. Puttock et al. (1998) developed the SCOPE 3 model (Shell Code for Overpressure Prediction in gas Explosions) to predict the overpressures which could be generated by gas explosions in vented enclosure and SCOPE 3 attempted, wherever possible, to model the underlying physical process in an explosion. Sklavounos et al. (2004) tested several turbulence models and compared them against experimental data of dense vapor dispersion, and computational results showed good agreement with experimental measurements indicating that CFDs provided a reliable means of estimating gas dispersion in real terrains. Van den Berg (1985) 
proposed a method for blast prediction which fully reflected characteristic features of vapor cloud explosions. Van den Berg et al. (1993) demonstrated that fundamental and practical objections were met if the TNT-equivalency concept is used for vapor cloud explosion hazard assessment, while the Multi-energy concept was shown to be a flexible concept which made it possible to incorporate current experimental data and advanced computational methods into the procedure of vapor cloud explosion hazard analysis. Leyer et al. (1993) proposed that the flame would accelerate when meeting the concentration gradient or turbulence and vortex caused by obstacles. Chen et al. (2009) studied the influence of obstacles on confined vapor cloud explosion by use of numerical simulation. Ma et al. (2014) investigated the impact of obstacles on gas explosion in tunnel. Qiao et al. (2010) quantified the potential overpressures due to vapor cloud explosions and the potential gas buildup by using CFD for onshore or offshore facilities. Tauseef et al. (2011) proposed a CFD-based method on basis of the finding that among the various models available for assessing turbulence, the realizable k- $\varepsilon$ model yields results closer to experimental findings than the other, more frequently used, turbulence models if used in conjunction with the eddy-dissipation model. Van Wingerden et al. (1999) demonstrated the application area of the FLACS method as well as the limitation of the traditional methods by comparing the CFD-method with traditional prediction methods being a TNT-equivalency method and the Multi-energy method for a practical case. In addition, the extent to which repeated obstacles are present ahead of any propagating premixed flame is also of major importance to the overpressures generated by explosions in many practical geometries. Harris et al. (1989) demonstrated that the presence of such obstacles could lead to increased flame speeds, and hence overpressure, through both flame distortion effects and the establishment of a feedback mechanism between burning rate and flow field turbulence, and also showed that the continued presence of repeated obstacles was required in order to maintain high flame speeds, with flames emerging from regions containing obstacles into unobstructed volumes decelerating rapidly. All of them found overpressure rose with the increase of VBR. However, when VBR was different, the number of obstacles also changed. So 
the VBR and the number of obstacles both resulted in that conclusion. Cong et al. (2008) carried out both acetylene experiment and numerical simulation and concluded that the smaller the obstacle voidage was and the more the number of obstacles was, the more powerful the gas explosion was. But in order to keep the single VBR, the gas volume becomes larger and larger with the increase of the number of obstacles. Previous researches on the numerical simulation of vapor cloud explosion have thought that the obstacle volume was negligible. So the obstacle volume was set to "Subgrid". However, actually, too many obstacles may occupy the space of gas, which may decrease the amount of combustible gas. On this occasion, the explosion power may be negatively affected.

This paper re-examined the influence of obstacles on the unconfined vapor cloud explosion and holds that obstacles could not only lead to the acceleration of flame, but also they may occupy some space, thus affecting the amount of combustible gas. A new method was raised to study the influence of obstacles. The influences of VBR and the number of obstacles on explosion power in opening congested environment are respectively studied using the AutoReaGas commercial code. Based on the numerical results of this study, the correlation between the explosion parameters and the obstacle structure has been obtained so that the explosion hazard effects at different obstacles structures can be acknowledged. The appropriate preventive and control measures of accidents for hydrogen and methane can be taken corresponding to the different obstacles structures. In addition, this study can provide a basis for the further numerical simulation studies of unconfined vapor cloud explosion.

\section{Computation Code and Governing Equations}

The commercial software package AutoReaGas, a finite element computational code for fluid dynamics suitable for gas explosion and blast problems, was used to carry out the numerical simulation process (Zhang et al., 2011). The code solves Navier-Stokes partial differential equations numerically by means of the finite volume formulation (Patankar, 1980). AutoReaGas was extensively validated against various experimental tests (Zhang et al., 2012; Ma et al., 2014; 
Zhang et al., 2011; Tufano et al., 1998). The gas dynamics used in the code is formulated as a set of conservation equations for mass, momentum and energy.

The conservation equation of mass is

$$
\frac{\partial \rho}{\partial t}+\frac{\partial\left(p u_{j}\right)}{\partial x_{j}}=0
$$

Where $\rho$ is the density; $t$ is the time; $p$ is the static pressure; $u$ is the flow velocity; $x$ is the space coordinate; $i, j$ is the coordinate direction.

The conservation equation of momentum is

$$
\begin{gathered}
\frac{\partial\left(\rho u_{i}\right)}{\partial t}+\frac{\partial\left(\rho u_{j} u_{i}\right)}{\partial x_{j}}=-\frac{\partial \rho}{\partial x_{i}}+\frac{\partial \tau_{i j}}{\partial x_{j}} \\
\tau_{i j}=\mu_{t}\left(\frac{\partial u_{i}}{\partial x_{j}}+\frac{\partial u_{j}}{\partial x_{i}}\right)-\frac{2}{3} \delta_{i j}\left(\rho k+\mu_{t} \frac{\partial u_{j}}{\partial x_{j}}\right)
\end{gathered}
$$

Where the turbulent viscosity coefficient $\mu_{t}=C_{\mu} \rho k^{2} / \varepsilon, k$ is the turbulent kinetic energy, $\varepsilon$ is the dissipation rate of turbulent kinetic energy, the model constant $C_{\mu}=0.09 ; \delta_{i j}$ is the Kronecker delta.

The conservation equation of energy is

$$
\frac{\partial(\rho E)}{\partial t}+\frac{\partial\left(\rho u_{j} E\right)}{\partial x_{j}}=\frac{\partial}{\partial x_{j}}\left(\Gamma_{E} \frac{\partial E}{\partial x_{j}}\right)-\frac{\partial\left(p u_{j}\right)}{\partial x_{j}}+\tau_{i j} \frac{\partial u_{i}}{\partial x_{j}}
$$

Where $\Gamma_{E}$ is the energy dissipation rate coefficient of turbulent flow; Specific internal energy $E=c_{V} T+m_{f u} H_{c}, \quad c_{V}$ is specific heat at constant volume; $T$ is the temperature; $m_{f u}$ is the fuel mass fraction; $H_{c}$ is the heat of combustion of fuel.

The conservation equation of fuel mass fraction is

$$
\frac{\partial\left(\rho m_{f u}\right)}{\partial t}+\frac{\partial\left(\rho u_{j} m_{f u}\right)}{\partial x_{j}}=\frac{\partial}{\partial x_{j}}\left(\Gamma_{f u} \frac{\partial m_{f u}}{\partial x_{j}}\right)+R_{f u}
$$

Where $R_{f u}$ is the volumetric combustion rate; $\Gamma_{f u}$ is the turbulent flow dissipation coefficient characteristic for fuel.

The turbulent flow field is modeled by a two-parameter $k-\varepsilon$ model, which uses conservation equations for the turbulence kinetic energy, $k$, and its dissipation rate, $\varepsilon$.

The turbulent flow kinetic energy equation is

$$
\frac{\partial(\rho k)}{\partial t}+\frac{\partial\left(\rho u_{i} k\right)}{\partial x_{j}}=\frac{\partial}{\partial x_{j}}\left(\Gamma_{k} \frac{\partial k}{\partial x_{j}}\right)+\tau_{i j} \frac{\partial u_{i}}{\partial x_{j}}-\rho \varepsilon
$$


Where $\Gamma_{k}$ is the turbulent flow dissipation coefficient characteristic of turbulent kinetic energy.

The dissipation rate of turbulent flow kinetic energy is

$$
\frac{\partial(\rho \varepsilon)}{\partial t}+\frac{\partial\left(\rho u_{j} \varepsilon\right)}{\partial x_{j}}=\frac{\partial}{\partial x_{j}}\left(\Gamma_{\varepsilon} \frac{\partial \varepsilon}{\partial x_{j}}\right)+C_{1} \frac{\varepsilon}{k} \tau_{i j} \frac{\partial u_{i}}{\partial x_{j}}-C_{2} \frac{\rho \varepsilon^{2}}{k}
$$

Where $\Gamma_{\varepsilon}$ is the turbulent flow dissipation coefficient characteristic for the dissipation rate of turbulent kinetic energy; $C_{1}$ and $C_{2}$ are the model constants.

The combustion reaction is considered as single step conversion from reactants to products, and the volume-based combustion rate $R_{c}$ has the following empirical expression:

$$
R_{c}=C_{t} \rho \frac{s_{t}^{2}}{\Gamma} R_{\min }
$$

Where $\Gamma$ is the turbulent diffusion coefficient; $R_{\min }$ is the minimum mass fraction among fuel, oxygen and products; $C_{t}$ is the dimensionless constant; $S_{t}$ is the turbulent burning velocity expressed through the Bray correlation

$$
S_{t}=1.8 u^{\prime 0.412} L_{t}^{0.196} S_{l}^{0.784} v^{-0.196}
$$

Where $u^{\prime}$ is the turbulent intensity; $L_{t}$ is the turbulent characteristic length scale; $S_{l}$ is the laminar burning velocity flammable mixture; $v$ is the kinematic viscosity of unburned mixture.

According to Eq. (8) and Eq. (9), the only physical mechanism of flame acceleration implemented in AutoReaGas is the burning rate enhancement due to the flow turbulence generated ahead of the flame front.

A quasi-laminar modification is used for the initial laminar combustion rate. The effects of pressure, temperature and flame front wrinkling on the laminar burning velocity are described by a second adjustable parameter $F_{s}$ which relates $S_{l, \text { eff }}$ to the flame radius $r$ and to the theoretical laminar flame speed:

$$
S_{l, e f f}=S_{l}\left(1+F_{s} r\right)
$$

The full set of constants used for the simulation is reported in Table 1.

Table 1 The set of constants used for the CFD simulations (AutoReaGAS User Manual Version $3.1,2002$ ) 


\begin{tabular}{cc}
\hline Constant & Value \\
\hline Combustion rate & \\
$\boldsymbol{F}_{\boldsymbol{s}}$ & 0.15 \\
$\boldsymbol{C}_{\boldsymbol{t}}$ & 160 \\
$\boldsymbol{k}-\boldsymbol{\varepsilon}$ model & \\
$\boldsymbol{C}_{\boldsymbol{\mu}}$ & 0.09 \\
$\boldsymbol{C}_{\boldsymbol{I}}$ & 1.44 \\
$\boldsymbol{C}_{2}$ & 1.79 \\
$\boldsymbol{\sigma}_{\boldsymbol{k}}$ & 1.0 \\
$\boldsymbol{\sigma}_{\varepsilon}$ & 1.3 \\
$\boldsymbol{F}_{\boldsymbol{k}}$ & 0.5 \\
\hline
\end{tabular}

\section{Verification of Numerical Method}

To verify the validity of the numerical method, the numerical calculation corresponding to the experimental conditions in previous study (Hansen et al., 2010) was conducted. The experiment is one of the series of MERGE project. The MERGE experiments (Mercx, 1994) were performed by British Gas (later Advantica and now Germanischer Lloyd) at the Spadeadam test site in the UK. The tests were financially supported by the European Commission, and were part of a larger project (Hansen et al., 2010).
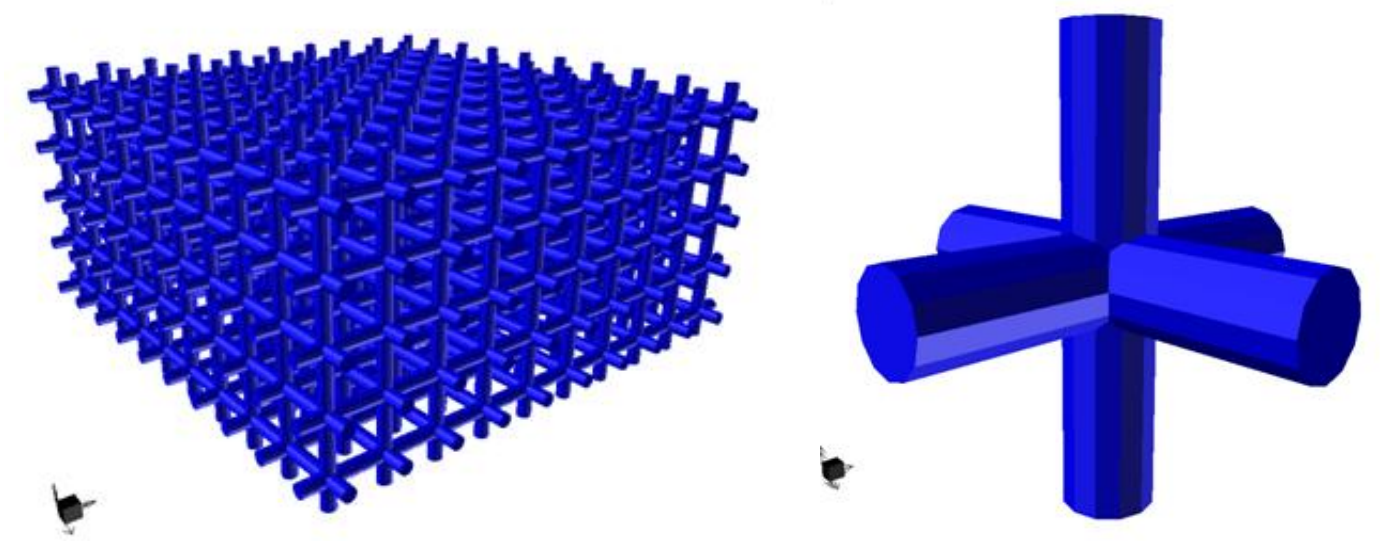

Fig. 1 Complete experimental rig and an element of obstacles array

Table 2 the detailed characteristics of these geometrics in the MERGE experiments

\begin{tabular}{cccc}
\hline Test & Pipes arrangement & Pipes diameter $/ \mathbf{~ c m ~}$ & Flammable scale $/ \mathbf{~ m}^{\mathbf{3}}$ \\
\hline MERGE-A & $20 \times 20 \times 10$ & 4.3 & 45 (medium) \\
MERGE-B & $30 \times 30 \times 15$ & 4.1 & 45 (medium) \\
MERGE-C & $10 \times 10 \times 5$ & 8.6 & 45 (medium) \\
MERGE-D & $16 \times 16 \times 8$ & 8.2 & 45 (medium) \\
MERGE-E & $10 \times 10 \times 5$ & 16.8 & 360 (large) \\
MERGE-C* & $20 \times 20 \times 10$ & 8.2 & 360 (large) \\
\hline
\end{tabular}


The MERGE tests were performed in six different experimental geometrics. Four cases including $\mathrm{A}, \mathrm{B}, \mathrm{C}$ and D were at medium scale of $45 \mathrm{~m}^{3}$ flammable cloud with $4 \mathrm{~m} \times 4 \mathrm{~m} \times 2 \mathrm{~m}$ obstruction array, whereas two cases E and $\mathrm{C}^{*}$ were at large scale of $360 \mathrm{~m}^{3}$ flammable cloud with $8 \mathrm{~m} \times 8 \mathrm{~m}$ x 4 m obstruction array. The detailed characteristics of these geometries were listed in Table 2 . The test $\mathrm{C}$ was chosen to verify the validity of the numerical method based on the appropriate medium scale and the arrangement and numbers of pipes. The test $\mathrm{C}$ was conducted in an enclosure measuring $4.5 \mathrm{~m} \times 4.5 \mathrm{~m} \times 2.25 \mathrm{~m}$ high surrounded by polythene sheeting that was kept rigidly in place at the edges for the medium-scale experiment, filled with methane premixed with air in stoichiometric ratio. Sufficient time had elapsed to ensure that the methane/air mixture was quiescent. The cube-shaped experiment region contained a regular pipe-work array (Cleaver et al., 1996). An example of the type of rig used is shown in Fig. 1. Obstacle array was formed from a number of circular cross-section tubes of $8.6 \mathrm{~cm}$ in diameter. Its type of arrangement is $10 \times 10 \times 5$ with a single spacing of $40 \mathrm{~cm}$ between pipes in each orthogonal direction. The ignition point was at ground level at the center of the congested region. All fuel/air mixtures were quiescent at the time of ignition and the methane/air mixture was ignited by a single, low energy spark (Cleaver et al., 1997). Several pressure transducers were located at ground level along the $\mathrm{x}$ axis direction. The distances to ignition source were $1.0 \mathrm{~m}, 1.2 \mathrm{~m}, 1.6 \mathrm{~m}, 2.0 \mathrm{~m}, 4.0 \mathrm{~m}$. All the experiments were conducted at atmospheric condition. In AutoReaGas simulations, the calculated scenario was almost the same as that in the experiment.

In numerical simulations, the pipes in the experiment were simplified as long cubic cylinders. The obstacles were set to "Solid". The structure is as shown in Fig. 2. The simulation was conducted in a $20 \mathrm{~m} \times 20 \mathrm{~m} \times 8 \mathrm{~m}$ area. The methane cloud was located at ground level at the center of the whole simulation region. The bottom of the model was set to a ground by selecting the "Solid" option, while others were open. The mesh was structured. Uniform mesh was adopted in the methane area and the grid cells were cubic. Adaptive mesh was adopted outside the methane area and the grid cell was cuboid. Two different numerical grids were adopted to verify the 
accuracy in the case of a large grid composed by 792000 cubic cells and a refined grid composed by 1176000 cubic cells. The element mesh of the large grid was $0.05 \mathrm{~m} \times 0.05 \mathrm{~m} \times 0.05 \mathrm{~m}$ in the methane area and the refined grid was $0.041 \mathrm{~m} \times 0.041 \mathrm{~m} \times 0.041 \mathrm{~m}$. The numerical results of these two types of grids were shown in Table 3. It appeared that the two computational results were consistent with each other as all the relative errors of peak overpressures were smaller than $8 \%$. Therefore, the grid size had almost no impact on the computational accuracy of peak overpressure. In order to save time and improve the computational efficiency, the large grid was adopted to perform all simulations in the following sections.

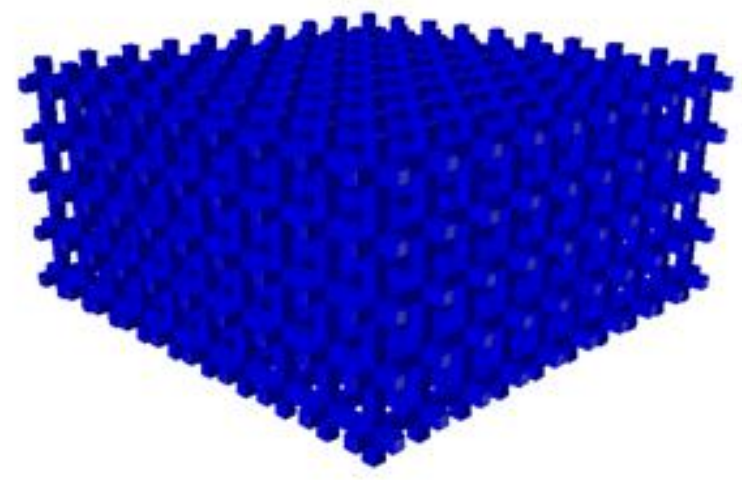

Fig. 2 Obstacles structure in numerical simulation Table 3 Peak overpressure of two types of grids $(\mathrm{kPa})$

\begin{tabular}{ccccc}
\hline \multirow{2}{*}{ Distance/m } & \multicolumn{2}{c}{ Grid number } & \multirow{2}{*}{ Absolute discrepancy } & Relative error/\% \\
\cline { 2 - 3 } & $\mathbf{7 9 2 0 0 0}$ & $\mathbf{1 1 7 6 0 0 0}$ & & 6.4 \\
\hline $\mathbf{1 . 0}$ & 10.336 & 9.672 & 0.664 & 6.7 \\
$\mathbf{1 . 2}$ & 10.353 & 9.655 & 0.698 & 6.7 \\
$\mathbf{1 . 6}$ & 10.357 & 9.658 & 0.699 & 7.8 \\
$\mathbf{2 . 0}$ & 10.225 & 9.431 & 0.794 & 3.5 \\
$\mathbf{4 . 0}$ & 6.856 & 6.619 & 0.237 & \\
\hline
\end{tabular}

The numerical simulation was compared with the experiment results as shown in Fig. 3. In Fig.

3, X-Distance is the distance to the ignition point along $\mathrm{x}$ axis direction. The explosion pressure in the experiment decreased along away from the ignition point. The pressures inside the obstacles region were obviously higher than those outside obstacles region and the maximum pressure was about $10.888 \mathrm{kPa}$. The pressure distribution of numerical simulation was similar with the 
experimental result. The maximum explosion pressure was about $10.357 \mathrm{kPa}$. As shown in Fig. 3, the peak overpressure of the numerical simulation agrees well with the experimental results totally. For quantitative comparison, the relative errors between simulated and experimental peak overpressure at various distances were shown in Table 4. The relative errors of most overpressure monitoring were less than $6 \%$. The experimental overpressure monitoring in an actual congested methane-air mixture explosion had some error itself. In addition, the environment including the wind speed had an impact on the experimental results. Therefore, the relative errors between the numerical simulation and the experimental results in Table 4 may result from both the experimental limitations and the numerical simulation.s

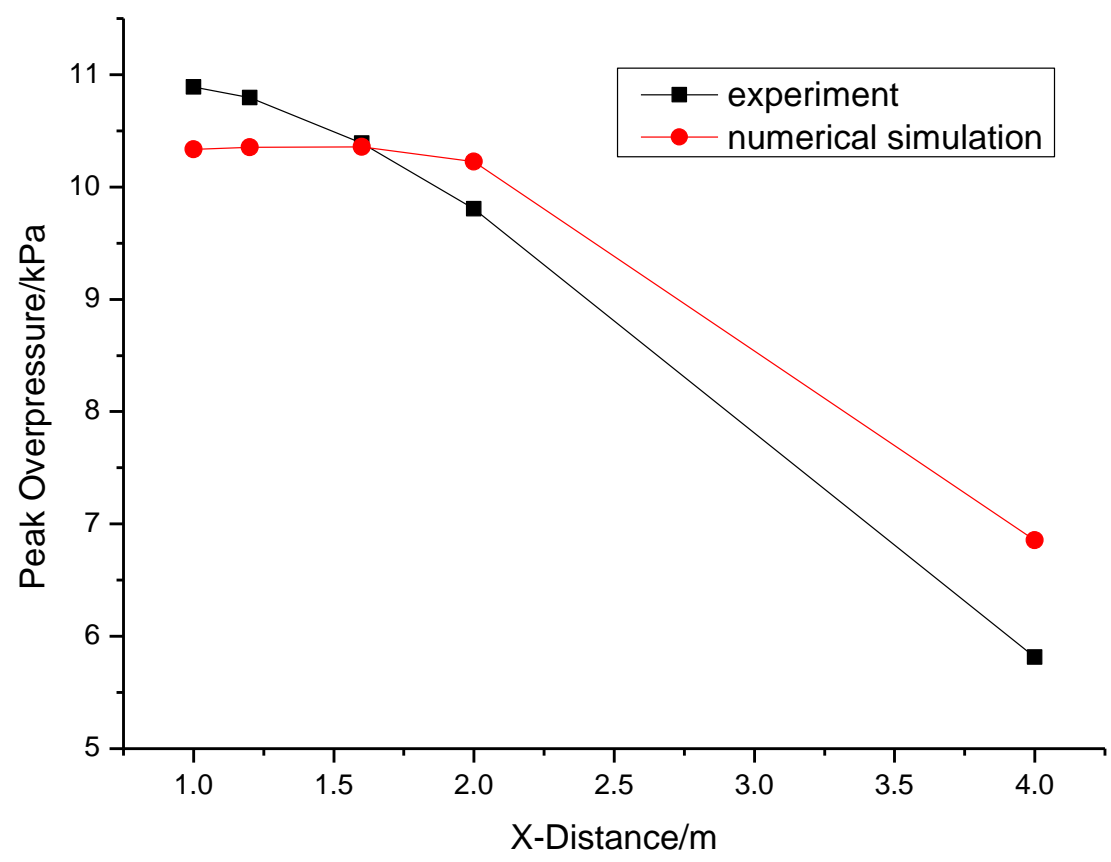

Fig. 3 Comparison of numerical simulation and experimental results

Table 4 Relative errors between simulated and experimental peak overpressures (Hansen et al., 2010) at various distances

\begin{tabular}{cccccc}
\hline X-Distance/ $\mathbf{m}$ & $\mathbf{1 . 0}$ & $\mathbf{1 . 2}$ & $\mathbf{1 . 6}$ & $\mathbf{2 . 0}$ & $\mathbf{4 . 0}$ \\
\hline Simulation value/ kPa & 10.336 & 10.353 & 10.357 & 10.225 & 6.856 \\
Experimental value/ kPa & 10.888 & 10.794 & 10.389 & 9.805 & 5.814 \\
Relative error/ \% & 5.07 & 4.09 & 0.31 & 4.28 & 17.92 \\
\hline
\end{tabular}


The above analysis and comparison verifies the validity of the numerical method and the code for gas explosion in actual congested vapor cloud.

\section{Simulation Scenarios}

The simulation was conducted in a $20 \mathrm{~m} \times 20 \mathrm{~m} \times 8 \mathrm{~m}$ area. The $32 \mathrm{~m}^{3}(4 \mathrm{~m} \times 4 \mathrm{~m} \times 2 \mathrm{~m})$ combustible gas cloud was located at ground level at the center of the whole simulation region. The combustible gas was a volume concentration of $4 \%$ for propane. Obstacles were set in the vapor cloud. Their structure is as shown in Fig. 4. The same method as above was adopted for meshing. The total number of numerical grid was 605000. The size of the grid cell of the combustible gas cloud was $0.05 \mathrm{~m} \times 0.05 \mathrm{~m} \times 0.05 \mathrm{~m}$. Thirty-two monitoring points were set evenly along $\mathrm{x}$-axis on the ground. The adjacent two monitoring points were at an interval of 0.3 m.

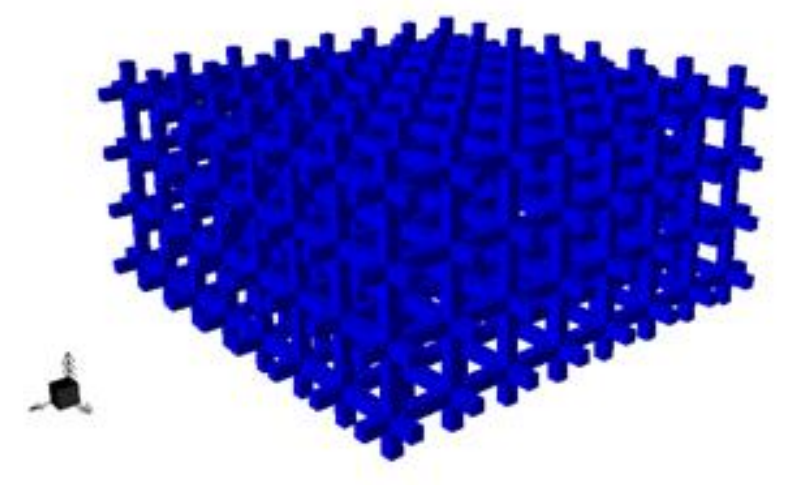

Fig. 4 Structure of obstacles in combustible gas cloud

In order to study the single influence of the VBR and the obstacles number on explosion parameters, a new two-step method was presented in the numerical simulation. In this method, when the influence of VBR was studied, the number and arrangement of obstacles were the same. Therefore, the influence of number and arrangement of obstacles could be avoided. Similarly, when the influence of number and arrangement of obstacles was studied, the VBR of obstacles was the same. Therefore, the effect of VBR could be eliminated. So this method could be used to study the influence of VBR and numbers of obstacles respectively. The simulation scheme is as shown in Table 5 and Table 6 . The distance below refers to the distance to the ignition point. 
Table 5 Structure parameters of various obstacle amounts for the VBR of 3.07

\begin{tabular}{cccc}
\hline $\begin{array}{c}\text { Obstacles } \\
\text { arrangement }\end{array}$ & Amount of obstacles & $\begin{array}{c}\text { Length of obstacle } \\
\text { cross section/ m }\end{array}$ & Spacing/ m \\
\hline $24 \times 24 \times 12$ & 1152 & 0.1 & 0.06 \\
$16 \times 16 \times 8$ & 512 & 0.15 & 0.1 \\
$12 \times 12 \times 6$ & 288 & 0.2 & 0.12 \\
$8 \times 8 \times 4$ & 128 & 0.3 & 0.2 \\
$4 \times 4 \times 2$ & 32 & 0.6 & 0.4 \\
\hline
\end{tabular}

Table 6 Parameters of obstacles under various VBRs for the obstacle arrangement of $8 \times 8 \times 4$

\begin{tabular}{ccc}
\hline VBR & $\begin{array}{c}\text { Length of obstacle cross } \\
\text { section/ } \mathbf{~}\end{array}$ & Spacing/ m \\
\hline 0.134 & 0.1 & 0.4 \\
0.348 & 0.15 & 0.35 \\
0.74 & 0.2 & 0.3 \\
1.54 & 0.25 & 0.25 \\
3.07 & 0.3 & 0.2 \\
\hline
\end{tabular}

\section{Results and Analysis}

5.1 Influence of the obstacle amount on explosion parameters for the given VBR

\subsubsection{Overpressure distribution with different amounts of obstacles}

Fig. 5 is a comparison and contrast of peak overpressure changing along the $\mathrm{x}$-axis under various amounts of obstacles when VBR is 3.07. Seeing from Fig. 5, it can be concluded that with the increase of the amount of obstacles, the peak overpressure rises gradually. The figure shows that the maximum peak overpressure was only 0.181Bar when the obstacles were arranged for $4 \mathrm{x}$ $4 \times 2$. While, when the obstacles arrangements were in $24 \times 24 \times 12$, the peak overpressure reach the highest value, up to 1.5 Bar. This is because that under the impetus of the flame front, the encounter of the obstacles by the unburned gas ahead of the flame may induce turbulence and greatly expand the flame surface, which can accelerate the turbulent transfer process for heat and material, thus promoting the combustion rate and increasing the explosion overpressure. The more 
obstacles are, the more the small openings are. The more intense turbulence is generated and makes combustion more acute. Therefore, the explosion overpressure is higher.

For the whole explosive field, the change trend of the peak overpressure is accordant for various amounts of obstacles. With the distance from the ignition point away, the peak overpressure decreases. This illustrates that the amount of obstacles do not affect the change trend of the peak overpressure in the whole explosion field.

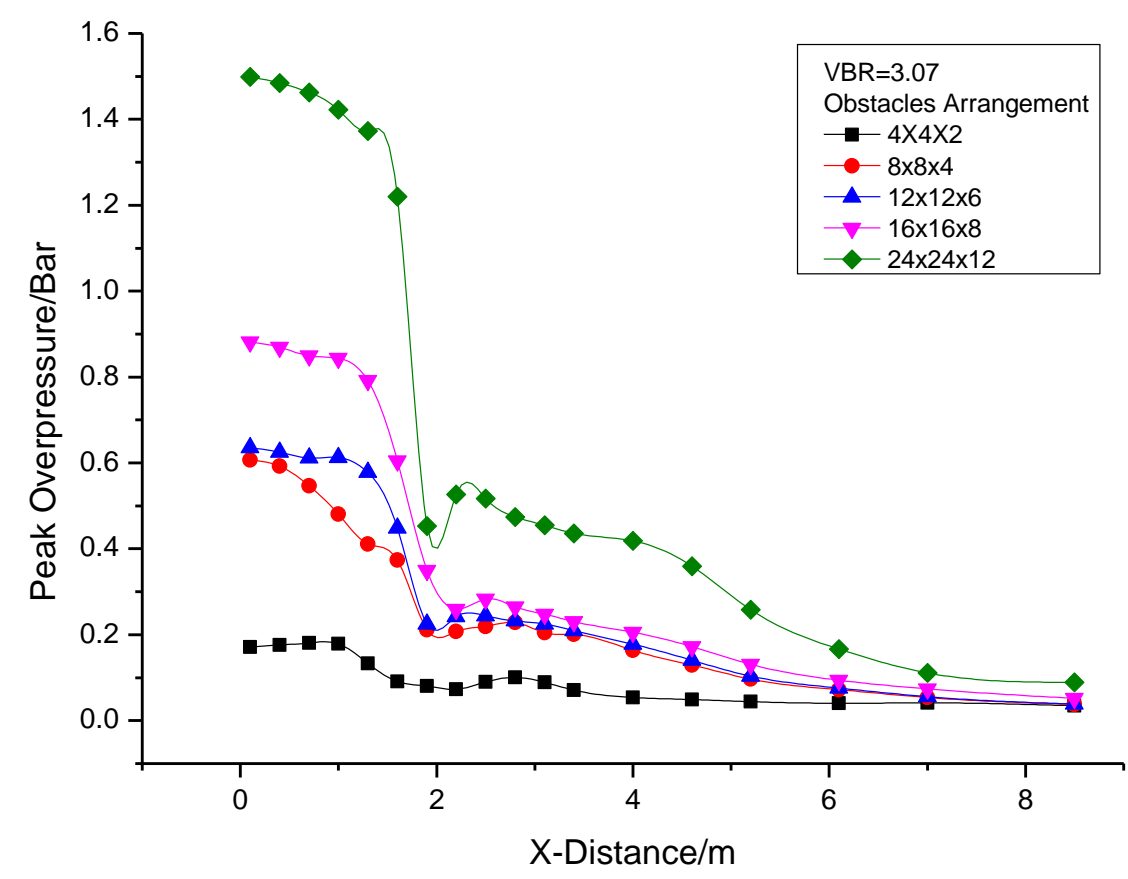

Fig. 5 Change trend of peak overpressure varying with number of obstacles along $\mathrm{x}$-axis direction 


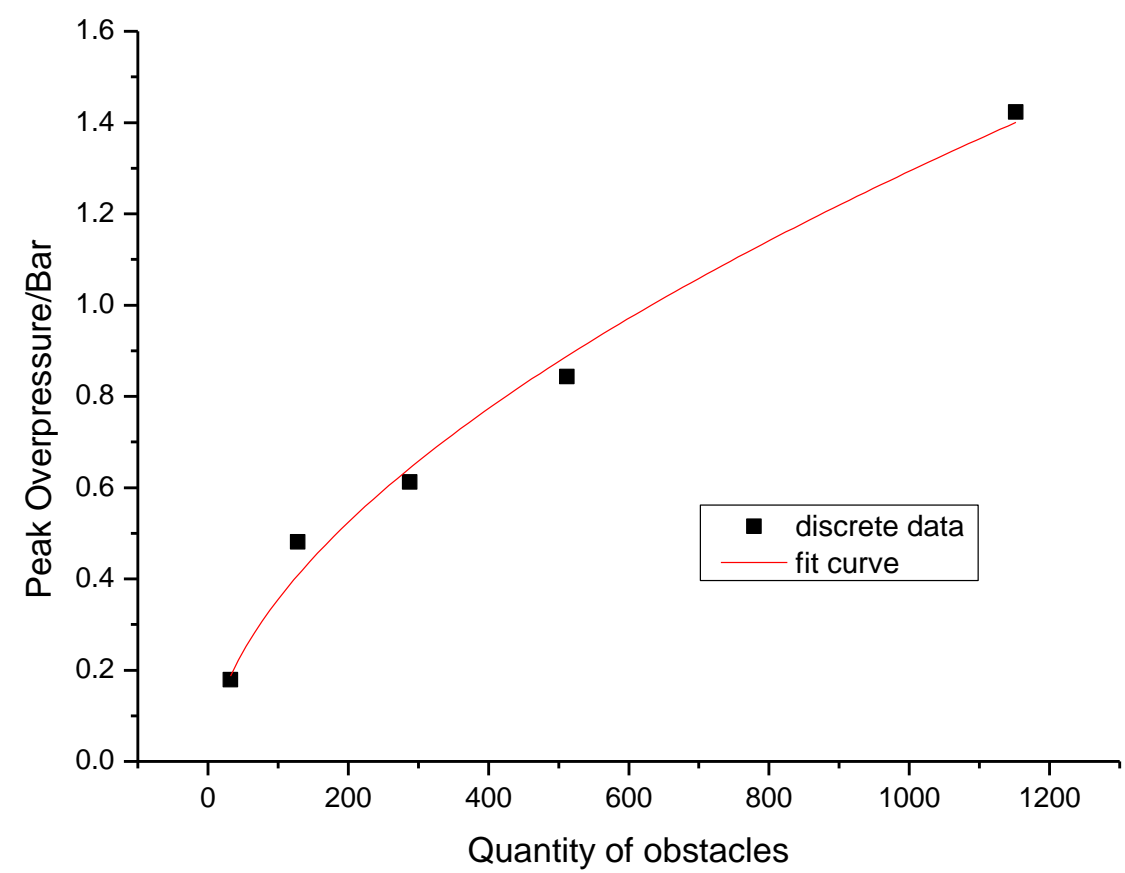

Fig. 6 Peak overpressure varying with number of obstacles

The peak overpressure curve is fitted based on the calculated results in order to predict the power of overpressure near the cloud boundaries for the different number of obstacles when VBR is 3.07. The correlation coefficient is 0.987 , which illustrates that the curve between the peak overpressure and the number of obstacles is well fitted. The quantitative relationship and fitting curve are given in Eq. (11) and Fig. 6.

$$
P_{\text {max }}=0.02675 n^{0.56144}
$$

Where $P_{\max }$ is the peak overpressure; $n$ is the total number of obstacles. Seeing from the equation, with the increase of the amount of obstacles, the peak overpressure is a power function of growth.

5.1.2 Change trend of combustion rate with the number of obstacles

The profile of the change trend of the maximum combustion rate with the number of obstacles along the $\mathrm{X}$-axis direction under a given VBR of 3.07 is shown in Fig. 7. In addition, the change 
trend of the maximum combustion rate without obstacle is also shown in Fig. 7. Due to the VBR is given, these obstacles are the same in volume, although the number of obstacles is varied. In addition, the amount of combustible gas is the same as well. Seeing from this profile, with the increase of the amount of obstacles, the maximum combustion rate is on the increase in the obstacle field. This is because as the amount of obstacles increases, the turbulence generated by the unburned gas is becoming stronger. In addition, the flame front area gradually also becomes larger. Therefore, the combustion gradually accelerates. Outside the obstacle field, the combustion rate decreases sharply when obstacles were arranged in $24 \times 24 \times 12,16 \times 16 \times 8,12 \times 12 \times 6$, but slowly when obstacles were arranged in $8 \times 8 \times 4,4 \times 4 \times 2$. Comparing with obstacle arrangement less dense than that in $8 \times 8 \times 4$, the combustion rate of propane is larger in the obstacle field when the obstacles are arranged in a denser way. There is more gas involved in the chemical reaction. The remaining little gas not involved in the chemical reaction diffuses outward. Near the original cloud border, the amount and the concentration of remaining gas decrease dramatically under the strong shock wave. Therefore, the combustion rate decreases sharply when obstacles are arranged in $24 \times 24 \times 12,16 \times 16 \times 8$, and $12 \times 12 \times 6$. However, when the obstacles are arranged in $8 \times 8 \times 4$, and $4 \times 4 \times 2$, the combustion rate of propane is lower in the obstacle field, and a portion of gas engages in the chemical reaction. There is a lot of gas not engaging in the chemical reaction remaining, and the amount and the concentration of remaining gas reduce slowly near the initial cloud border. So the corresponding combustion rate reduces slowly. 


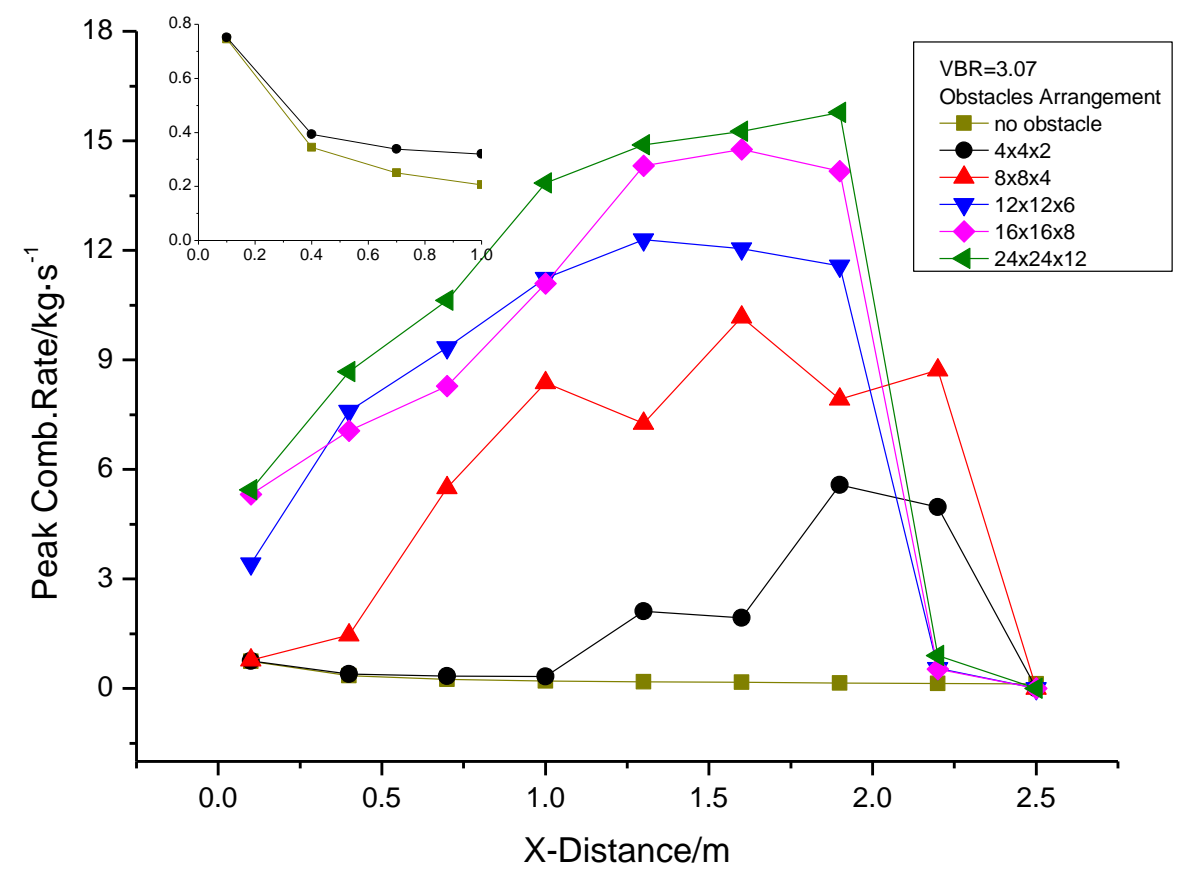

Fig. 7 Change trend of maximum combustion rate with number of obstacles along $\mathrm{X}$-axis direction

5.2 Influence of VBR on explosion parameters for given number of obstacles

\subsubsection{Overpressure distribution with different VBRs}

The change trend of the peak overpressure with different VBRs along the $\mathrm{x}$-axis direction under a given obstacle arrangement of $8 \times 8 \times 4$ is presented in Fig. 8 . The peak overpressure is up firstly and then down as the VBR increases. The peak overpressure reaches the maximum at the VBR of 0.74 and the maximum value is $0.628 \mathrm{Bar}$. As the VBR increases, the volume of obstacles becomes larger and larger. Thus, the amount of combustible gas reduces correspondingly. The accelerating effect of obstacles on flame is greater than the influence of combustible gas amount when VBR is less than 0.74 . The obstacles promote the propagation of flame and accelerate the gas combustion. Therefore, the overpressure gradually rises. When VBR is greater than 0.74 , the influence of combustible gas amount plays a leading role. Greater VBR means larger obstacles volume. And the combustible gas is less correspondingly. So the obstacles occupy too much space so that the 
combustible gas is too little. And the turbulence generated by combustible gas is relatively weak. Meanwhile, the energy released also reduces dramatically. The effect of flame acceleration weakens. Thus, the overpressure gradually goes down.

The change trend of peak overpressure for different VBRs is consistent with that for different amounts of obstacles. With the distance from the ignition point away, the peak overpressure goes down. It illustrates that the amount and volume of obstacles have little influence on the change trend of overpressure.

The peak overpressure curve is fitted based on the calculating results in order to predict the power of overpressure near the cloud boundaries for different VBRs when the obstacles are arranged in $8 \times 8 \times 4$. Seeing from the curve, the correlation coefficient is 0.96 , which illustrates that the curve between the peak overpressure and VBR is well fitted. The quantitative relationship and fitting curve are given in Eq. (12) and Fig. 9.

$$
P_{\text {max }}=0.419+\frac{0.408 e^{-M}}{0.707 \sqrt{2 \pi} V B R}
$$

Where VBR is the obstacle volume blockage ratio; $M=\left(\ln \frac{\mathrm{VBR}}{1.404}\right)^{2}$ 


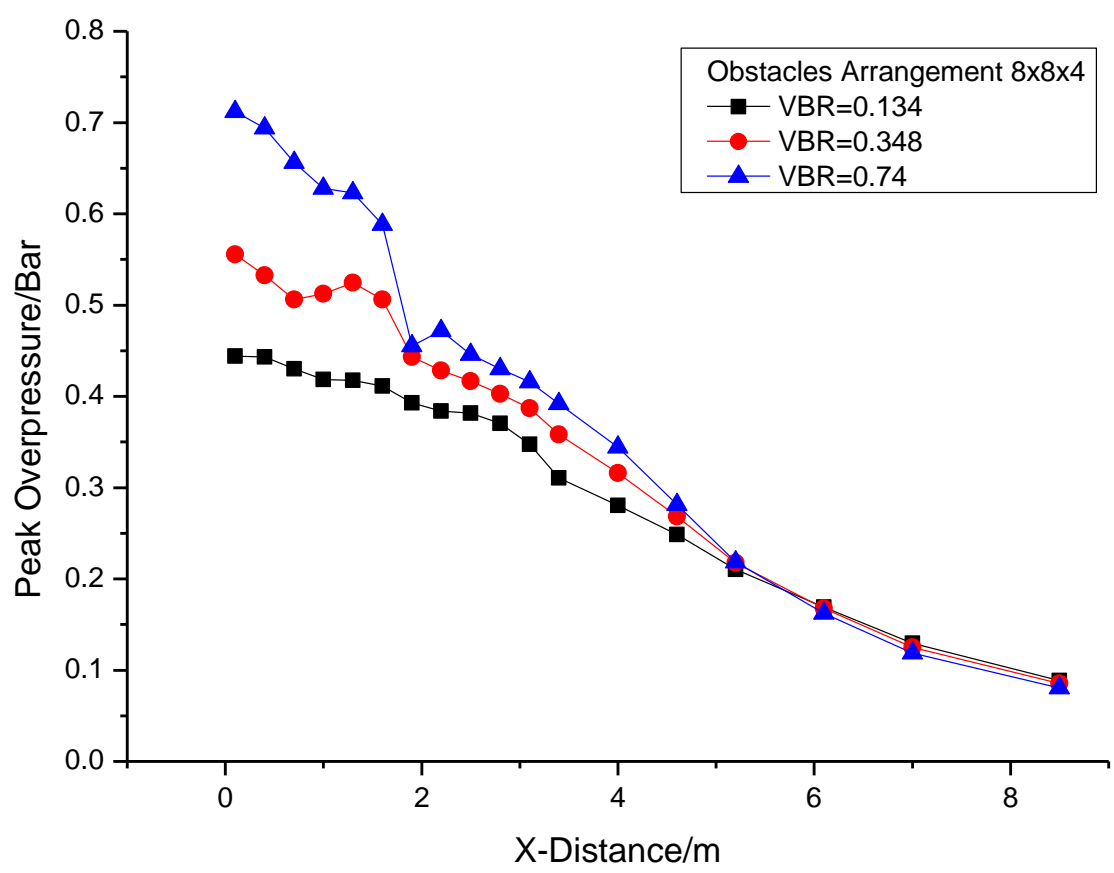

(a) $\mathrm{VBR}=0.134 、 0.348 、 0.74$

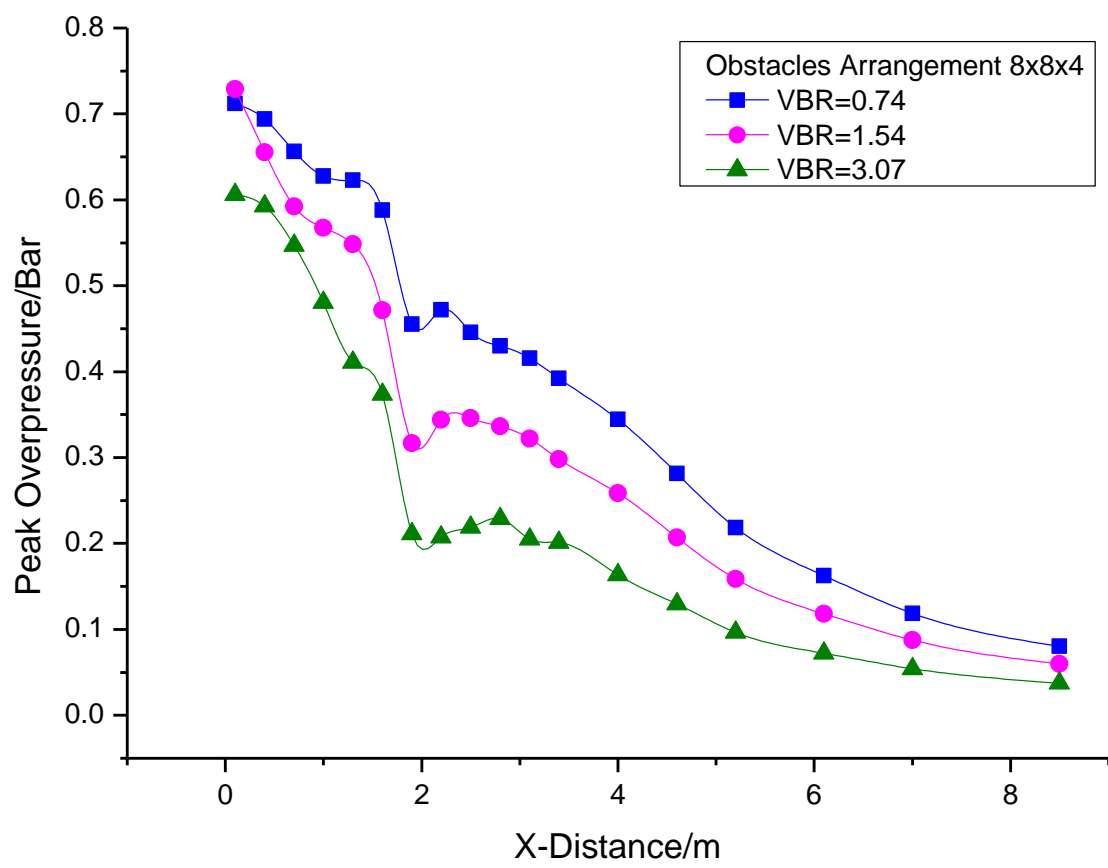

(b) $\mathrm{VBR}=0.74 、 1.54 、 3.07$

Fig. 8 Change trend of peak overpressure varying with VBR along $\mathrm{x}$-axis direction 


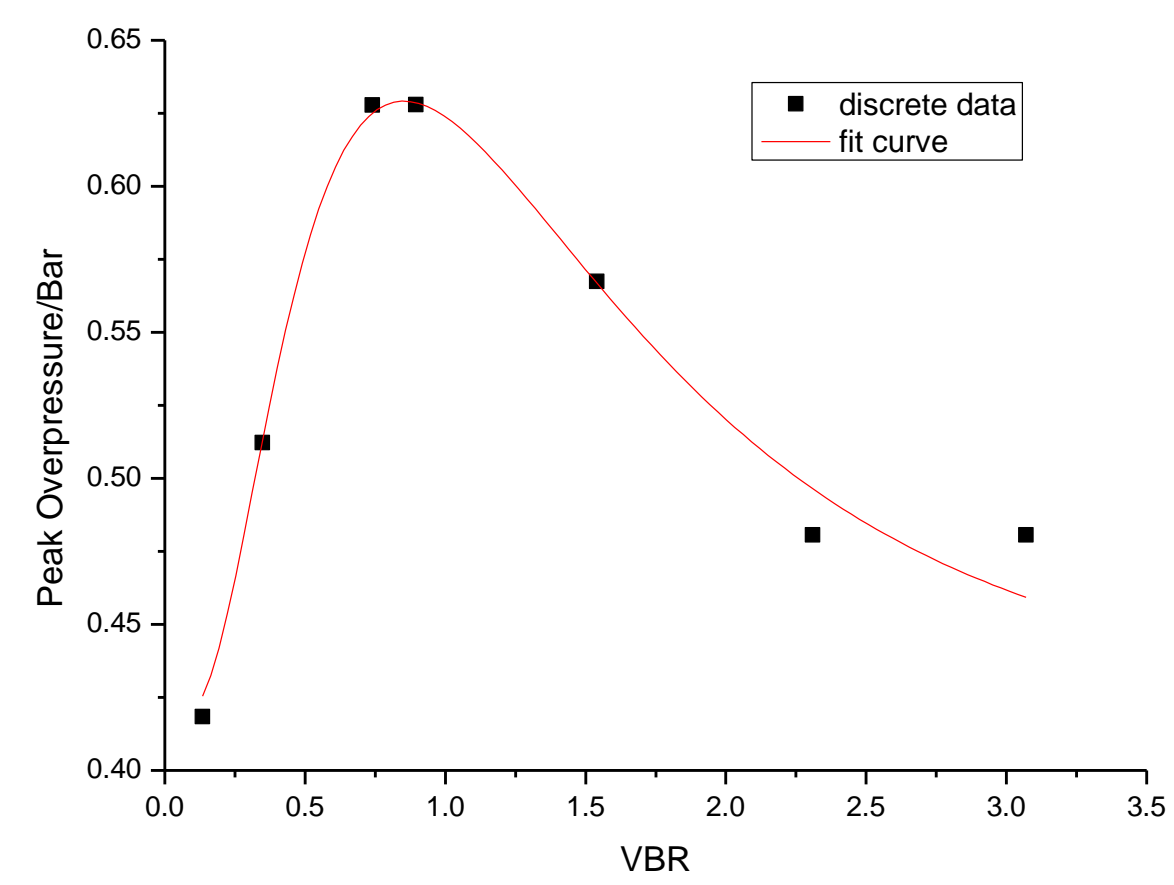

Fig. 9 Peak overpressure varying with VBR

\subsubsection{Change trend of combustion rate with VBR}

The change trend of the maximum combustion rate with VBR along the $\mathrm{x}$-axis direction when obstacles are arranged in $8 \times 8 \times 4$ is shown in Fig. 10. In addition, the change trend of the maximum combustion rate without obstacle is also displayed in Fig. 10. Similar with peak overpressure, the maximum combustion rate is up firstly and then down with increase of VBR. When VBR is up to 0.74 , the peak overpressure reaches the highest. When VBR is less than 0.74 , the accelerating effect of obstacles on flame is greater than that of combustible gas volume. The combustion rate rises gradually with VBR. When VBR exceeds 0.74 , the inhibitory effect of combustible gas volume plays a leading role. Thus, the combustion rate is down slowly as VBR increases. 


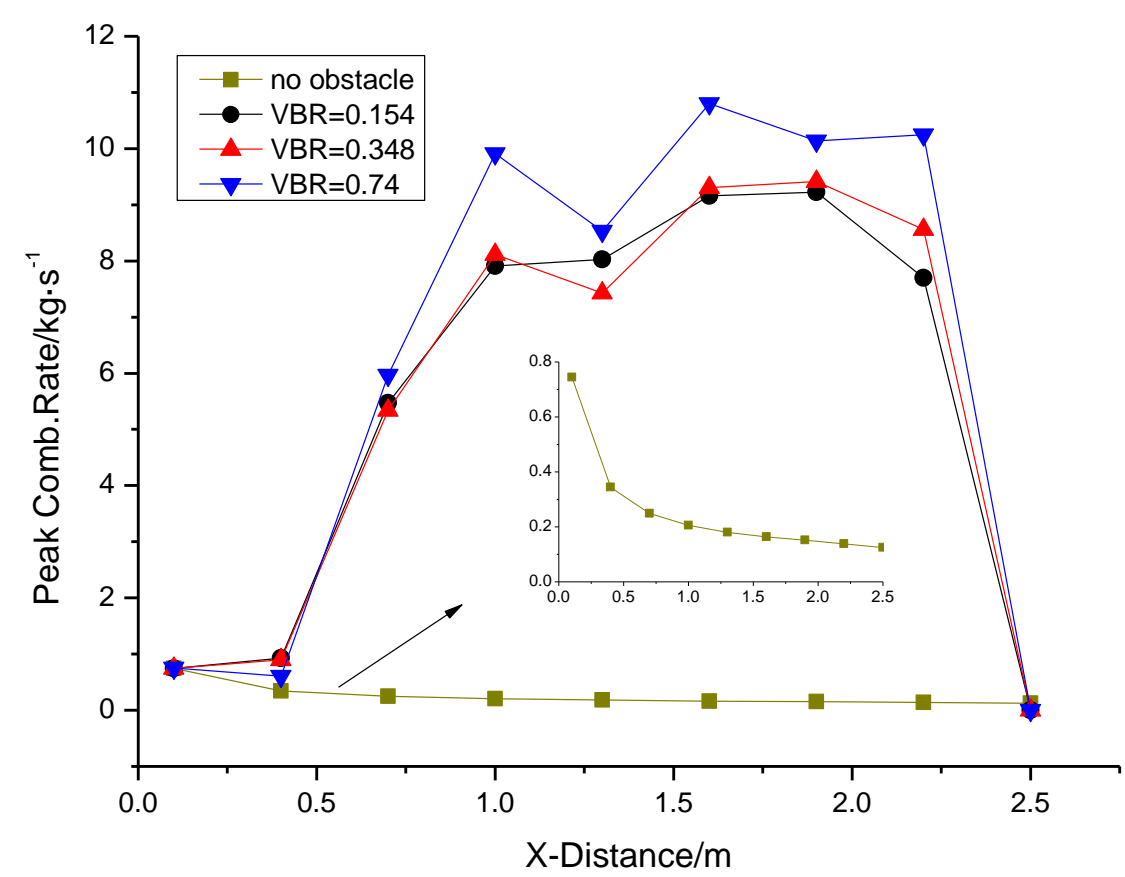

(a) No obstacles and VBR=0.134, 0.348, 0.74

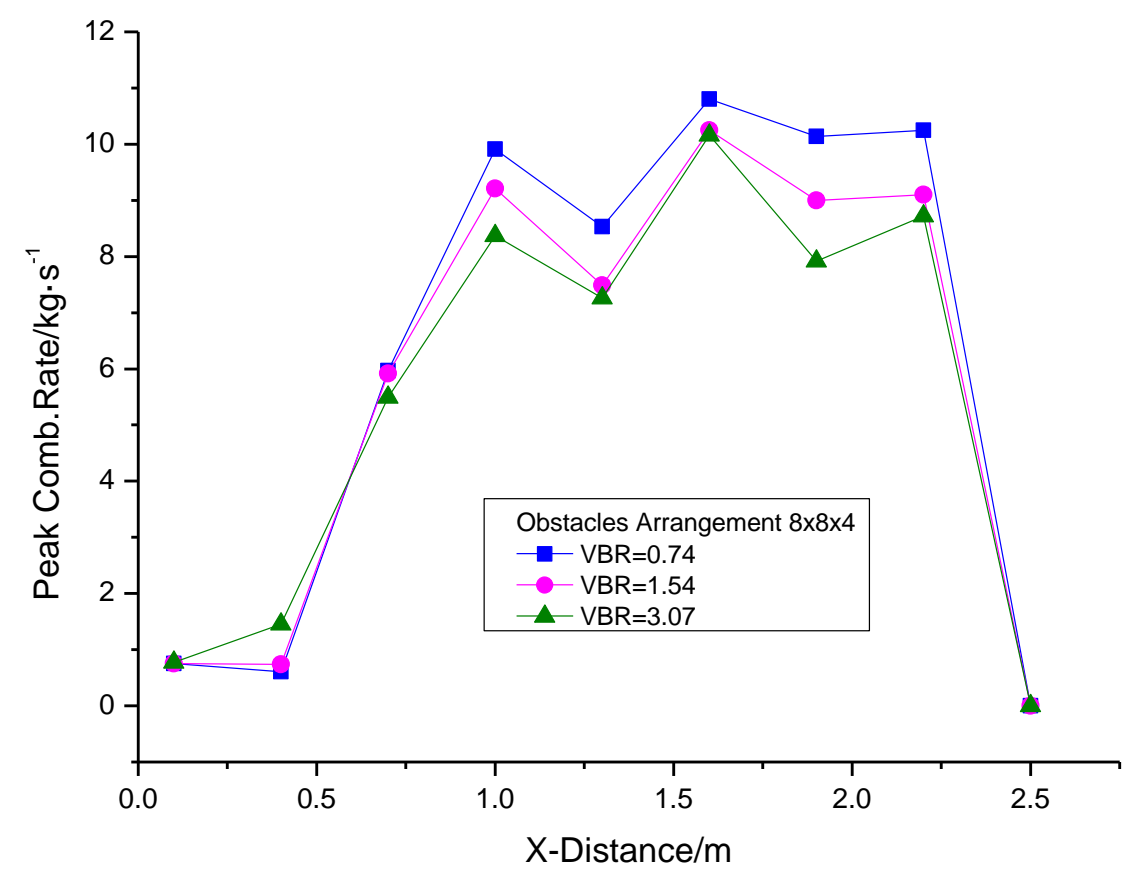

(b) $\mathrm{VBR}=0.74,1.54,3.07$

Fig. 10 Change trend of maximum combustion rate varying with VBR along $\mathrm{X}$-axis direction In addition, seeing from Fig. 7 and Fig. 10, the combustion rate reduces to 0 near a distance of 2.5 meters from ignition point. This shows that whatever the number and VBR of obstacles are, 
the fireball radius is about 2.5 meters. Therefore, it is concluded that the existence and structure of obstacles have little effect on the fireball size when the combustible gas clouds are the same in size and concentration.

\section{Conclusion}

The obstacle structure in the vapor cloud has a significant influence on the gas explosion Obstacles could not only lead to the acceleration of flame, but also they may occupy some space, thus affecting the amount of combustible gas. In this paper, a new two-step method was proposed to respectively study the effects of the obstacles amount and volume blockage ratio (VBR) on the gas explosion by using Computation Fluid Dynamic software AutoReaGas, and the obstacles in the vapor cloud were set to "Solid" instead of "Subgrid". Based on the results and analysis, the main conclusions can be described in the following points.

(1) Under a given VBR, the peak overpressure and the maximum combustion rate of unconfined propane/air cloud explosion rise with the number of obstacles. The more the obstacles are, the higher the gas explosion parameters are. The maximum peak overpressure was about 0.181 Bar for the obstacles arrangement of $4 \times 4 \times 2$ and reaches 1.5 Bar for the obstacles arrangement of $24 \times 24 \times 12$. These were indicated that the vapor cloud explosion of more obstacles was more dangerous and more destructive for a single VBR.

(2) Under a given number of obstacles, the peak overpressure and the maximum combustion rate of unconfined propane/air cloud explosion are up firstly and then down with increase of VBR and reach the highest value of 0.628 Bar at the VBR of 0.74 , which indicated that the intensity of vapor cloud explosion reach a peak at a certain VBR in the middle instead of the largest.

(3) The existence and structure of obstacles have little effect on the size of explosion fireball 
when the size and concentration of combustible gas cloud are given. When the size of vapor cloud was $4 \mathrm{~m} \times 4 \mathrm{~m} \times 2 \mathrm{~m}$ and the concentration is $4 \%$, the radius of explosion fireball was about $2.5 \mathrm{~m}$.

\section{Acknowledgments}

The research presents in this paper was supported by the National Science Foundation of China (11372044).

\section{References}

Arntzen, B.J., 1998. Modelling of turbulence and combustion for simulation of gas explosions in complex geometries. PhD thesis: Norwegian University of Science and Technology, Trondheim.

AutoReaGas User Manual Version 3.1., 2002. AutoReaGAS User Manual Version 3.1. England: Century Dyamics and TNO.

Bjerketvedt, D., Bakke, J.R., \& Wingerden, K.V., 1997. Gas explosion handbook. J. Hazard. Mater. 57, 1-150.

Chen, W.Y., \& Chai, J.S., 2009. Numerical simulation of explosion overpressure field of flammable vapor with barriers in open space. China Safety Science Journal, 19, 35-40.

Cleaver, R.P., Humphreys, C.E., Morgan, J.D., \& Robinson, C.G., 1997. Development of a model to predict the effects of explosions in compact congested regions. J. Hazard. Mater. 53, 35-55.

Cleaver, R.P., \& Robinson, C.G., 1996. An analysis of the mechanisms of overpressure generation in vapor cloud explosions. J. Hazard. Mater. 45, 27-44.

Cong, L.X., \& Bi, M.S., 2008. Effects of obstructions on impulse of flammable gas cloud deflagrations. J. Loss Prevention Process Ind. 21, 118-123.

Hansen, O.R., Hinze, P., Engel, D., \& Davis, S., 2010. Using computational fluid dynamics (CFD) for blast wave predictions. J. Loss Prevention Process Ind. 23, 885-906.

Harris, R.J., \& Wickens, M.J., 1989. Understanding vapor cloud explosions-an experimental study. In 55th autumn meeting of the institution of gas engineers (27281989). 
Leyer, J.C., \& Desbordes, D., 1993. Unconfined Deflagrative Explosion without Turbulence: Experiment and Model. J. Hazard. Mater. 34, 123-150.

Ma, Q.J., Zhang, Q., \& Pang, L., 2014. Effects of hydrogen addition on the confined and vented explosion behavior of methane in air. J. Loss Prevention Process Ind. 27, 65-73.

Ma, Q.J., Zhang, Q., \& Pang, L., 2014. Influence of the tunnel wall surface condition on the methane-air explosion. Combust. Explos. Shock Waves 50, 208-213.

Mercx, W.P.M., 1994. Modeling and experimental research into gas explosions. Overall report of the MERGE project, CEC contract: STEP-CT-0111(SSMA).

Patankar, S.V., 1980. Numerical Heat Transfer and Fluid Flow. Hemisphere Publishing Corporation, Washington, DC.

Puttock, J.S., Yardley, M.R., \& Creswell, T.M., 1998. Prediction of vapor cloud explosions using the SCOPE model. J. Loss Prevention Process Ind. 13, 419-431.

Qiao, A., \& Zhang, S., 2010. Advanced CFD modeling on vapor dispersion and vapor cloud explosion. J. Loss Prevention Process Ind. 23, 843-848.

Sklavounos, S., \& Rigas, F., 2004. Validation of turbulence models in heavy gas dispersion over obstacles. J. Hazard. Mater. 108 (1-2), 9-20.

Tauseef, S.M., Rashtchian, D., Abbasi, T., Abbasi, S.A., 2011. A method for simulation of vapor cloud explosions based on computational fluid dynamics (CFD). J. Loss Prevention Process Ind. 24, 638-647.

Tufano, V., Maremonti M., Salzano E., \& Russo G., 1998. Simulation of VCEs by CFD modeling: an analysis of sensitivity. J. Loss Prevention Process Ind. 11, 169-175.

Van den Berg, A.C., 1985. The multi-energy method: a framework for vapor cloud explosion blast prediction. J. Hazard. Mater. 12, 1-10.

Van den Berg, A.C., \& Lannoy, A., 1993. Methods for vapor cloud explosion blast modeling. J. Hazard. Mater. 34, $151-171$

Van Wingerden, K., Hansen, O.R., \& Foisselon, P., 1999. Predicting blast overpressures caused by vapor cloud explosions in the vicinity of control rooms. Process Saf. Prog. 18 (1), 17-24. 
Zhang, Q., Pang, L., \& Liang, H.M., 2012. Coupling relation between air shockwave and high-temperature flow from explosion of methane in air. Flow, Turbul. Combust. 89, 1-12.

Zhang, Q., Pang, L., \& Liang, H.M., 2011. Effect of scale on the explosion of methane in air and its shockwave. J. Loss Prevention Process Ind. 24, 43-48.

Zhang, Q., Pang, L., \& Zhang, S.X., 2011. Effect of scale on flame speeds of methane-air. J. Loss Prevention Process Ind. 24, 705-712. 
Table 1 The set of constants used for the CFD simulations (AutoReaGAS User Manual Version $3.1,2002)$

\begin{tabular}{cc}
\hline Constant & Value \\
\hline Combustion rate & \\
$\boldsymbol{F}_{s}$ & 0.15 \\
$\boldsymbol{C}_{\boldsymbol{t}}$ & 160 \\
$\boldsymbol{k}-\varepsilon$ model & \\
$\boldsymbol{C}_{\boldsymbol{\mu}}$ & 0.09 \\
$\boldsymbol{C}_{\boldsymbol{I}}$ & 1.44 \\
$\boldsymbol{C}_{2}$ & 1.79 \\
$\boldsymbol{\sigma}_{\boldsymbol{k}}$ & 1.0 \\
$\boldsymbol{\sigma}_{\varepsilon}$ & 1.3 \\
$\boldsymbol{F}_{\boldsymbol{k}}$ & 0.5 \\
\hline
\end{tabular}


Table 2 the detailed characteristics of these geometrics in the MERGE experiments

\begin{tabular}{cccc}
\hline Test & Pipes arrangement & Pipes diameter $/ \mathbf{c m}$ & Flammable scale $/ \mathbf{~ m}^{\mathbf{3}}$ \\
\hline MERGE-A & $20 \times 20 \times 10$ & 4.3 & 45 (medium) \\
MERGE-B & $30 \times 30 \times 15$ & 4.1 & 45 (medium) \\
MERGE-C & $10 \times 10 \times 5$ & 8.6 & 45 (medium) \\
MERGE-D & $16 \times 16 \times 8$ & 8.2 & 45 (medium) \\
MERGE-E & $10 \times 10 \times 5$ & 16.8 & 360 (large) \\
MERGE-C* & $20 \times 20 \times 10$ & 8.2 & 360 (large) \\
\hline
\end{tabular}


Table 3 Peak overpressure of two types of grids $(\mathrm{kPa})$

\begin{tabular}{ccccc}
\hline \multirow{2}{*}{ Distance/m } & \multicolumn{2}{c}{ Grid number } & \multirow{2}{*}{ Absolute discrepancy } & \multirow{2}{*}{ Relative error/\% } \\
\cline { 2 - 3 } & $\mathbf{7 9 2 0 0 0}$ & $\mathbf{1 1 7 6 0 0 0}$ & & 6.4 \\
\hline $\mathbf{1 . 0}$ & 10.336 & 9.672 & 0.664 & 6.7 \\
$\mathbf{1 . 2}$ & 10.353 & 9.655 & 0.698 & 6.7 \\
$\mathbf{1 . 6}$ & 10.357 & 9.658 & 0.699 & 7.8 \\
$\mathbf{2 . 0}$ & 10.225 & 9.431 & 0.794 & 3.5 \\
$\mathbf{4 . 0}$ & 6.856 & 6.619 & 0.237 & \\
\hline
\end{tabular}


Table 4 Relative errors between simulated and experimental peak overpressures(Hansen et al., 2010) at various distances

\begin{tabular}{cccccc}
\hline X-Distance/ $\mathbf{~}$ & $\mathbf{1 . 0}$ & $\mathbf{1 . 2}$ & $\mathbf{1 . 6}$ & $\mathbf{2 . 0}$ & $\mathbf{4 . 0}$ \\
\hline Simulation value/ $\mathbf{k P a}$ & 10.336 & 10.353 & 10.357 & 10.225 & 6.856 \\
Experimental value/ $\mathbf{k P a}$ & 10.888 & 10.794 & 10.389 & 9.805 & 5.814 \\
Relative error/ \% & 5.07 & 4.09 & 0.31 & 4.28 & 17.92 \\
\hline
\end{tabular}


Table 2 Structure parameters of various obstacle amounts for the VBR of 3.07

\begin{tabular}{cccc}
\hline $\begin{array}{c}\text { Obstacles } \\
\text { arrangement }\end{array}$ & Amount of obstacles & $\begin{array}{c}\text { Length of obstacle } \\
\text { cross section/ m }\end{array}$ & Spacing/ m \\
\hline $24 \times 24 \times 12$ & 1152 & 0.1 & 0.06 \\
$16 \times 16 \times 8$ & 512 & 0.15 & 0.1 \\
$12 \times 12 \times 6$ & 288 & 0.2 & 0.12 \\
$8 \times 8 \times 4$ & 128 & 0.3 & 0.2 \\
$4 \times 4 \times 2$ & 32 & 0.6 & 0.4 \\
\hline
\end{tabular}


Table 3 Parameters of obstacles under various VBRs for the obstacle arrangement of $8 \times 8 \times 4$

\begin{tabular}{ccc}
\hline VBR & $\begin{array}{c}\text { Length of obstacle cross } \\
\text { section/ } \mathbf{~}\end{array}$ & Spacing/ m \\
\hline 0.134 & 0.1 & 0.4 \\
0.348 & 0.15 & 0.35 \\
0.74 & 0.2 & 0.3 \\
1.54 & 0.25 & 0.25 \\
3.07 & 0.3 & 0.2 \\
\hline
\end{tabular}


Graphical Abstract
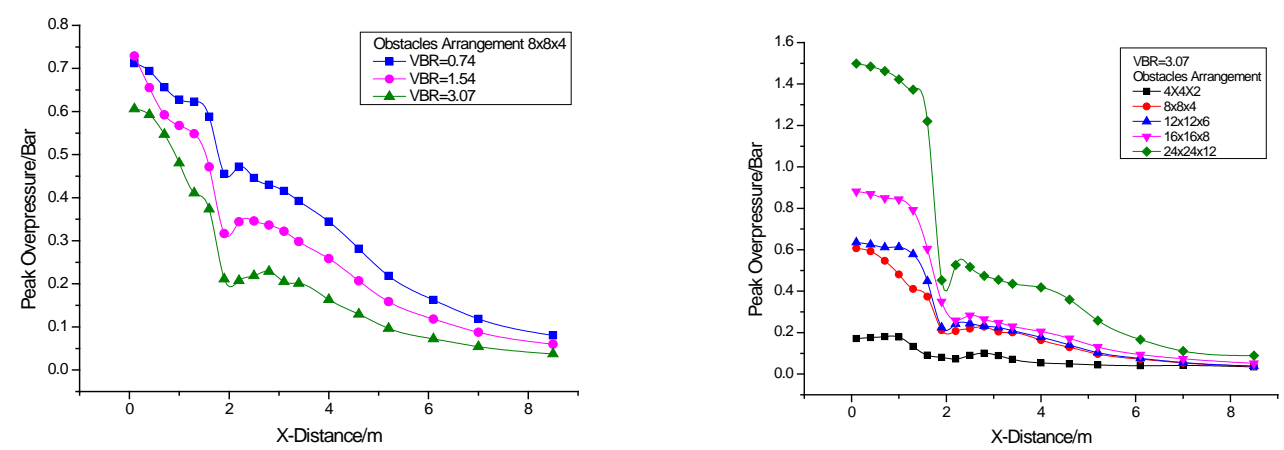